\title{
6. Über die Polarisation in der Geißlerentladung $\left.{ }^{1}\right)$; von Erich Rumpf:
}

1. Vor einiger Zeit ist mir der Nachweis gelungen, daB an Quersonden eines GeiBlerrohres Erscheinungen auftreten, die eine grobe Ähnlichkeit mit elektrolytischer Polarisation aufweisen; bei ihrer Besprechung verwendete ich die Ausdrücke "Anfangsströme, Dauerströme, Rủckgänge und Eigenströme". Ich habe meine damalige Veröffentlichung als einen vorläufigen Bericht bezeichnet und habe betont, daB noch verschiedene Fragen ungeklärt erschienen.

Nun habe ich die Versuche an der durch Fig. 1 (a.a. O.) wiedergegebenen Anordnung fortgesetzt und fand alle seinerzeit gemachten Erfahrungen bestätigt. Damit decken sich auch Versuche, die Ernst Reiche ${ }^{8}$ ) gelegentlich einer Arbeit „Über den Beginn der Glimmentladung in Luft" veröffentlicht hat, die mir bei Besprechung der Literatur entgangen war.

Ich habe damals vorgeschlagen, diese Polarisationserscheinungen durch kondensatorartige Oberflächenschichten zu erklären, vermied es aber, auf den Vorgang näher einzugehen. Nun will ich versuchen, nachzuweisen, dak die beschriebenen Polarisationserscheinungen tatsächlich durch Oberflächenschichten bedingt sind. Solche Oberflächenschichten werden ja in der physikalischen Praxis mehrfach beobachtet. Z. B. führt auch Reiche die von ihm beobachteten "störenden Nebenerscheinungen“ auf „elektrische Doppelschichten durch Gasionen, die in der Gashaut der Elektroden stecken bleiben" zurück und Robert $\mathrm{Pohl}^{3}$ ) spricht von "elektrisch geladenen, polarisationsS. 1.1910 .

1) Als AbscbluB meiner gleichnamigen Arbeit: Ann. d. Phys. 59.

2) E. Reiche, Ann. d. Phys. б2. S. 109. 1917.

3) R. Pohl, Die Physik der Röntgenstrahlen. Die Wissenschaft. Heft 45. Vieweg, Braunschweig 1912. 
ähnlichen Oberfächenschichten" gelegentlich einer Besprechung von Versuchen von 0. v. Bayer und A. Tool. ${ }^{1}$ )

2. Wenn die von mir beschriebenen Polarisationserscheinungen durch kondensatorartige Schichten, die die Elektroden umgeben, verursacht wurden, so muBte sich ein ballistisches Galvanometer ausgezeichnet zu ihrer Beobachtung eignen. Ich habe daher ein empindliches Galvanometer $G^{\prime}$ von hoher Schwingungsdauer in meine Versuchsanordnung so eingefugt, daß es durch die Eigenströme der Sonden in Schwingungen versetzt werden konnte. Hierfür boten sich vornehmlich zwei Stellen, die beide gleich gute Wirkung hatten: entweder statt des Elster- und Geitelschen Elektrometers am Schalter $H$

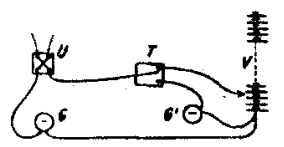

Fig. 1.

(Fig. 1, a. a. 0.) oder hinter dem Taster.T in der Weise, wie durch die beigegebene Fig. 1 ersichtlich ist, die die genannte Schaltungsskizze ergänzt; beim Niederdrücken des Tasters $T$ waren in diesem Falle die Galvanometer $G$ und $G^{\prime}$ hintereinandergeschaltet, während der Dauerstrom nur durch das Galvanometer $G$ flieBen kounte. Meist kam diese Schaltung in Verwendung.

Als ballistisches Galvanometer stand mir ein Drehspuleninstrument von Hartmann und Braun zur Verfügung mit etwa 17 Sekunden (ganzer) Schwingungsdauer; der Widerstand ist mit $10000 \mathrm{Ohm}$ angegeben, die zweite Systemspule von $5 \mathrm{Ohm}$ war durch einen gleich großen Widerstand geschlossen, wodurch eine bequeme Dämpfung erreicht wurde.

3. Im folgenden will ich kurz über die Ergebnisse berichten, die mit dieser erweiterten Versuchsanordnung gefunden wurden; und zwar haben sich die Untersuchungen hauptsächlich auf die Abhängigkeit der ballistischen Ausschläge von der erregenden Spannung $V$ (Fig. 1) und von dem Obertlächenzustande der Sonden erstreckt.

Wenn an die Sonden eine Spannung $V$ angelegt wurde, so konnte im Galvanometer $G$ AnfangsstromstoB und Dauerstrom abgelesen werden, wie dies a. a. O. durch die Figg. 2, 3,

.1) O. v. Bayer und A. Tool, Verhandl. d. Deutscb. Physik. Ges. 13. S. 569. 1911. 
5 und 6 beschrieben ist. Wurde darauf durch Niederdrücken des Tasters $T$ (Fig. 1) die Batterie abgeschaltet und gleichzeitig das Sondenpaar mit den beiden nunmehr hintereinandergeschalteten Galvanometern $G$ und $G^{\prime}$ unmittelbar verbunden, so zeigten im allgemeinen beide Galvanometer Ausschläge, nach denen sie sofort in die Ruhelage zuruckkehrten: $G$ aperiodisch, $G^{\prime}$ nach wenigen Schwingungen. Die Ausschläge von $G$ folgten den a. a. O. durch Fig. 4 und 7 dargestellten Regeln und waren dort als Eigenströme bezeichnet worden; die Ausschläge von $G^{\prime}$ zeigten einen Verlauf, der an der Hand der folgenden Fig. 2 beschrieben werden wird.

Vollkommen stellten sich allerdings die Ruhelagen nach diesen Ausschlägen meist nicht ein, da die Sonden meist ein verschiedenes Kontaktpotential haben und sich dann wohl auch lichtelektrisch so verschieden verhalten, daß sie infolge der heftigen lichtelektrischen Erregung durch die GeiBlerentladung Ströme aussenden, die besonders die Stellung des empfindlicheren Galvanometers $G^{\prime}$ wesentlich beeinflussen.

Inshesondere sind diese Ruhelagestörungen sehr groB, wenn die Sonden in absichtlich verschiedenem Zustande untersucht wurden: z. B. die eine frisch geschmirgelt, die andere alt, $d . h$. im Entladungsrohr wiederholt mit Strom belastet. Dann sind die Ruhelagestörungen oft so grob, daB sie die GröBenordnung der ballistischen Ausschläge erreichen, weshalb von einer Untersuchung solcher Sondenzusammenstellungen Abstand genommen werden muBte. Daher konnte ich mit dieser Anordnung auch nicht in Erfahrung bringen, welche von den Elektroden, die kathodische oder die anodische, den Hauptanteil an der kondensatorartigen Wirkung trägt, wie es a. a. O. geschehen war, denn die Ruhelagestörungen sind im unempfindlich geschalteten Galvanometer $G$ viel weniger zu merken. Es müssen aber natürlich die a. a. O. geschlossenen Folgerungen aufrecht bleiben, wie ich besonders in Punkt 6 zeigen werde.

Die Erscheinungen waren auch diesmal an allen untersuchten Metallen (Kupfer, Zink und Platin-Iridium) ganz äbnlich.

4. Eingehend habe ich namentlich ein paar Zinksonden untersucht, die $1 \mathrm{~mm}$ dick waren und $6 \mathrm{~cm}$ aus der Glimmer- 
scheibe (a. a. O., S. 6) herausragten. Der Entladungsstrom $J$ im Rohr betrug bei diesen Versuchen im Mittel 1,3 Milliamperes, das Rohr war, mit verdunnter Luft vom Drucke $0,3 \mathrm{~mm} \mathrm{Hg}$ gefüllt; die Sonden waren $7 \mathrm{~cm}$ von der Kathode entfernt, befanden sich also im auslaufenden Teil des negativen Glimmlichtes. Diese Verhältnisse sind für die Beobachtung insofern sehr günstig, als hier eine starke Drehung der Sonden aus ihrer horizontalen Lage nötig ist, um infolge des Feldes im Geiblerrohr einen merklichen Ausschlag in den Galvanometern bzw. im Elektrometer zu erhalten.

In der beigegebenen Fig. 2 sind aus einer großen Anzahl von gesammelten Kurren drei herausgegriffene dargestellt, wobei die Mittel aus den Werten eingezeichnet sind, die man

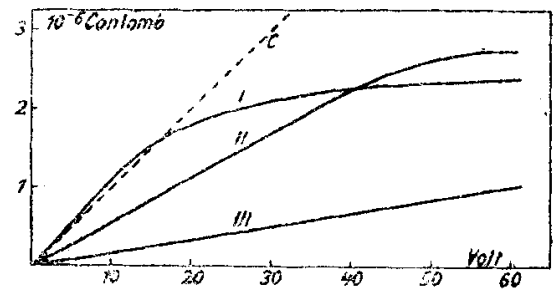

Fig. 2 .

erhält, wenn man die Sonden einmal in der einen und dann in der anderen Richtung mit Strom von der Batterie $V$ belastet. Dadurch sind störende Nebenerscheinungen, wie solche durch das immer etwas verschiedene Kontalktpotential der Sonden entstehen, unterdrückt worden. Die dazugehörigeu Anfangs- bzw. Dauerströme zeigen im Vergleich mit den a. a. 0 , Figg. 2, 3, 5 und 6 abgebildeten nichts Neues und sollen daher hier nicht neu besprochen werden. Als Abszissen sind in der obenstehenden Fig. 2 die an die Sonden angelegten Spannungen $V$ in Folt aufgetragen, als Ordinaten die in Mikrocoulomb umgerechneten ballistischen Ausschläge des Galvanometers $G^{\prime}$. Zur Eichung wurden statt der Sonden an die Zuleitungsdrähte ein Glimmerkondensator von 0,1 Mikrofarad (Elli iot, London) angelegt, wodurch die Gerade $C$ erhalten wurde.

Die Kurve I wurde von Sonden gewonnen, die vor der Beobachtung geschmirgelt worden waren; die Kurve steigt an- 
fänglich angenähert linear an, biegt dann um und nähert sich einem konstanten Wert, der trotz weiterer Erhöhung der Spannung nicht überschritten werden kann. Die Sondenkapazität kann in diesem Zustande der Sonden keine wesentlich höhere Elektrizitätsmasse fassen, als etwa 21/2 Mikrocoulomb; da diese Kurve in ihrem ansteigenden Teil der Goraden $C$ nahe liegt, erkennt man, daB dieser 'T'eil ungefähr' einem Kondensator von $1 / 10$ Mikrofarad entspricht.

Die Kurve II ist das Ergebnis von Beobachtungen, die mit denselben Sonden angestellt wurden, nachdem sie durch etwa 4 Stunden, ron den Leitungen abgeschaltet und untereinander kurz geschlossen, dem Entladungsstrom $J$ ausgesetzt waren. Durch diese Behandlung ist der linear ansteigende T'eil länger und deutlicher ausgeprägt geworden und entspricht, wio ein Vergleich mit der Geraden $C$ ergibt, einer Sondenkapazitit von etwa ${ }^{1 / 20}$ Mikrofarad; gleichzeitig ist jener 'Teil der Kurve, der sich der Horizontalen nähert, höher gerückt: die Sondenkapazität ist in diesem Zustande fähig, eine etway höhere Elektrizitätsmenge zu fassen, als ror der vierstündigen Behandlung im Rohr.

Nachdern nunmehr die Sonden in gleicher Weise wie vorher durch etwa 20 Stunden der GeiBlerentladung ausgesetzt worden waren, wurde die Kurve III gewonnen, bei der nur mehr der ansteigende Teil ausgebildet ist, der einer Sondenkapazität von $1 / 60$ Mikrofarad entspricht.

Eine weitere Erhöhung der Spannung ist nicht tunlich, weil dann leicht lichtbogenartige „Funkenstöße“ zwischen den Sonden überspringen, die nicht nur das Galvanometer $G$ gefährden, sondern auch die Oberflächenbeschaffenheit der Sonden sofort derart verïndern, daB der Kurventypus ganz anders wird, z. B. von III nach I überspringt. Solche FunkenstöBe treten bei neuen Sonden (I) schon bei viel geringeren Spannungen auf, als bei alten (III), wo oft die zur Verfügung stehende Spannung von 120 Volt nicht ausreichte, um FunkenstöBe zu erzeugen, während sie bei ganz frischen Sonden auch schon unter 60 Volt auftraten.

5. Werden die Sonden, nachdem sie ihre Ladungen durch die Galvanometer ausgeglichen haben, durch Umlegen des 
Schalters $H$ (a. a. O., Fig. 1) an das Elektrometer angeschlossen, das nun so eingestellt war, daB 40 Skalenteile 2 Volt entsprachen, so zeigt dieses anfänglich keinen Ausschlag. Nach einigen Sekunden beginnt es aber eine Potentialdifferenz anzuzeigen, die der vorhergegangenen erregenden Spannung $V$ entgegengerichtet ist; der Ausschlag wächst in weniger als einer Minute bis zu einem gewissen Wert an, um dann wieder langsam zu fallen. Der so erreichte gröBto Ausschlag ist abhängig von der vorhergegangenen Erregung der Sonden; er ist bei kleineren Erregungsspaunungen geringer, bei stärkeren gröBer, nähert sich aber mit steigender Erregungsspannung einem Endwert, der meist kleiner ist als 2 Volt, oder diesen Wert nicht weit überschreitet.

Es ist, als ob sich an den Sondenkapazitäten schwache Restladungen ausbilden würden. Vielleicht hat man es hier auch mit einer nachhaltigen Variation des Kontaktpotentiales zu tun, mit einer Polarisation im engeren Sinne; in diesem Falle könnte diese Erscheinung mit der Erholung eines erschöpften Akkumulators verglichen werden.

Sie steht aber im Vergleich mit den übrigen hier auftretenden "Polarisationserscheinungen" an Stärke weit zurück and wurde nicht näher untersucht.

6. Es ist nun die Frage zu beantworten, wieso die beiden Galvanometer $G$ und $G^{\prime}$ scheinbar ganz verschiedene Resultate geben: $G$ zeigt die "Eigenströme" an, wie a. a. O. durch die Figg. 4 und 7 dargestellt wurde, während $G^{\prime}$ ballistische Ausschläge und damit Elektrizitätsmengen anzeigt, die der Fig. 2 entsprechen.

Da muB zunächst festgestellt werden, daB der Ausdruck "Eigenströme", der a. a. O. zur leichteren Beschreibung eingeführt worden war, nicht sehr glücklich gewählt scheint, denn es handelt sich hier um kurzdauernde Entladungen, die wesentlich unter der Schwingungsdauer der Galvanometer liegen, wit aus verschiedenen Versuchen gefolgert werden kounte. Es sind mithin die Bedingungen für Anwendung eines ballistischen Galvanometers gegeben.

Diese Entladungen erfolgen im Augenblick des Niederdrückens des Tasters $T$ gleichzeitig mit dem Aussetzen des 
Dauerstromes. Sie treiben also das Galvanometer $G^{\prime}$ aus der Ruhelage, während sie im aperiodisch geschalteten Galvanometer $G$ eine Geschwindigkeit $u_{0}$ bewirken, die die Rückkehrgeschwindigkeit der Galvanometerspule nach dem Aussetzen des dem Dauerstrom entsprechenden Dauerausschlages in die Ruhelage vergrößert. Die Durchrechnung der Bewegung des aperiodischen Galvanometers ergibt nun, daB nur dann Ausschläge über die Ruhelage hinaus - die den Eigenströmen entsprechen würden - entstehen könren, wenn $u_{0}$ gröBer ist als diejenige Geschwindigkeit $U$, mit der die Galvanomete:spule ungedämpft aus dem Dauerausschlag durch die Ruhelage schwingen würde. $U$ ist nun proportional dem Daueratusschlag (ähnlich Figg. 2, 3, 5 und 6, a. a. 0.$), u_{0}$ proportional dem ballistischen Ausschlag (Fig. 2); aus dem Vergleich zweier solcher zusammengehöriger Kurvenbilder ergibt sich, daß die mit "Eigenstrom" bezeichneten Galvanometerausschläge mit steigender Spannung $V$ einen Verlauf zeigen müssen, der eiu Maximum hat, wie dies tatsächlich gefunden und a. a. 0 . an der Hand der Figg. 4 und 7 beschrieben wurde. Umgekehrt lann man aus den Eigenströmen mit ihren a. a. O. beschrie. benen GesetzmäBigkeiten ebeuso wie aus den Rückgängen, für die natürlich die obigo Überlegung auch gilt, ohne Schwierigkeit auf Entladungen schlieBen, die den durch die Fig. 2 wiedergegehenen GesetzmäBigkeiten entsprechen.

Leider konnte ich keine erfolgversprechende Methode austindig machen, um die den Anfangsströmen, bzw. den Rückgängen entsprechenden Elektrizitätsmengen unmittelbar ballistisch aufzunehmen. Es dürften aber die hier gemachten Angaben genügen, um die kondensatorartige Wirkung der Sondenoberfächen zu erweisen und die Erscheinungen in befriedigender Weise zu deuten.

7. Sehr leicht gelingt der Nachweiz, daB es sich tatsächlich um Vorgänge handeln muB, die an den Oberflächen der Sonden haften, also um oberfächliche Schichten und nicht etwa um räumliche Gobilde im Innern des Gases zwischen den Sonden, wenn man etwa folgenden Versuch anstellt: Durch das Entladungsrohr fliebe der Strom $J$, der Schalter $H$ sei in der Galvanometerstellung, der 'laster $T$ in seiner oberen Lage, von 
der Batterie $V$ sei eine beliebige Spannung angeschaltet; dann zeigt $G$ den Dauerstrom und $G^{\prime}$ steht auf Noll. Wenn nun $J$ unterbrochen wird, verschwindet natürlich die GeiBlerentladung und es kehrt $G$ ohne Schwingung in die Nullage zurück. Drthckt man hierauf $T$ in seine untere Lage, so bleiben beide Galvanometer vollkommen ruhig (wenn sich keine Isolationsfehler eingeschlichen haben!). Wird nun $J$ wieder geschlossen, so zeigen beide Galvanometer Ausschläge ${ }^{1}$ ), die in beiden Instrumenten sofort wieder verschwinden, in $G$ aperiodisch, in $G^{\prime}$ nach wenigen Schwingungen. Es ist wohl nicht nötig, zu sagen, daB ich keine Ausschläge bekommen konnte, wenn die Spannung $V$ gleich Null war.

Auffällig war bei solchen Versuchen, daB zwischen dem Unterbrechen und dem abermaligen SchlieBen des Entladungsstromes lange Zeiten verstreichen konnten - nach einer halben Stunde wurde noch keine sonderliche Verminderung der ballistischen Ausschläge beobachtet. ${ }^{2}$ ) Die kondensatorartigen Schichten halten also im stromlosen Rohr sehr gut ihre Ladungen, während bei geschlossenem $J$ schon wenige Sekunden nach dem Abschalten ron $V$ ein merklicher Abfall in den Galvanometerausschlägen zu bemerken ist, weil sich dann die Ladungen durch das ionisierte Gas ausgleichen.

Aus solchen Versuchen scheint mir einwandfrei hervor. zugehen, $\mathrm{daB}$ es sich hier nicht um räumliche Schichten im Entladungsrohr handelt, sondern tatsächlich um Vorgänge, die sich an den Oberflächen der Sonden abspielen. Ubrigens führen sowohl die a. a. $O$. auf S. 11 beschriebenen Leuchterscheinungen, als auch der mehrfach erörterte EinfluB des Oberflächenzustandes zu diesem SchluB.

8. Unter Annahme von kondensatorartigen Gashäuten oder elektrischen Doppelschichten an den Sondenoberflächen gestatten die beschriebenen Beobachtungen folgende Deutung: Solche Schichten bilden sich - wieso, möge später besprochen werden - sehr rasch nach dem Schmirgeln oder Schaben der Sonden, haben aber dann nur eine geringe Widerstandsfestig-

1) Hierbei gehen beide Galvanometer parallel, d. h. $G$ zeigt nun mit steigendem $V$ keine Maxima, sondern einen Verlauf wie Fig. 2 mit entsprechend geringerer Empfindlichkeit.

2) O. v. Bayer und A. Tool (a. a. O.) fanden nach 8 Tagen deutlicho Oberflichenladungen bei ibren Versuchen. 
keit: sie sind nur imstande, eine geringe Elektrizitätsmenge zu tragen. Wird diese durch Erhöhung der Spannung über ein bestimmtes $\mathrm{MaB}$ erhöht, so dringen die Ionen oder Eelektronen durch die trennende Haut hindurch, wodurch ein stärkerer Ausgleich der Ladungen stattfindet, während bei geringerer Spannung die Leitfähigkeit der Schichten geringer ist.

Die Sondenkapazitäten sind zu vergleichen mit Kondensatoren mit parallel geschalteten Widerständen, deren Größe abhängig ist von der angelegten Spannung. Ist diese gering, so ist die Leitfähigkeit des Aggregates verhältnismäBig gering, wird die Spannung größer, so fällt der Widerstand. Im nichtionisierten Gas, bei Unterbrechung des GeiBlerstromes ist auch die Jeitfähigkeit anf Null gesunken, weshalb die Sondenkapazitäten ihre Ladungen in so ausgezeichneter Weise halten. Die Leitfähigkeit der Schichten ist also gebunden an die Ionisation im Rohr.

Daraus möchte ich schließen, daB die Leitfähigkeit der Schichten hervorgerufen wird durch Elektronen, die durch die Schichten hindurchdringen können, während die größeren Ionen in den Schichten bleiben. Diese Elektronen werden einesteils durch die StoBionisation in der Gasentladung selbst geliefert und wandern zur anodischen Sonde, anderenteils durch lichtelektrische Erregung des Metalls der kathodischen Sonde innerhalb der Gashaut. Nach dieser Hypothese wäre noch das Durchdringungsvermögen abhängig von der Schichtdicke und von der treibenden Potentialdifferenz. Mit den Erscheinungeu nicht unvereinbar wäre noch die weitere Annahme, daB bei höheren Potentialdifferenzen, im horizontalen Teil der Kurven, auch die Ionen ein gewisses Durchdringungsvermögen erlangen.

Die GeiBlerentladung, der die Sonden vor der Aufnahme der Kurre II ausgesetzt waren, bewirkt ein Anwachsen der Hautdicke. Dadurch wird die Sondenkapazität geringer, weil der Abstand der Kondensatorbelege größer geworden ist; aber trotzdem rermag sie eine höhere Elektrizitätsmenge zu fassen, weil die Festigkeit der Gashaut gewachsen ist, d. h. die dickeren Schichten dem Hindurchdringen der Elektronen ein stärkeres Hindernis entgegensetzen. Hierdurch ist der (im Modell parallel geschaltete) Sondenwiderstand gestiegen, was auch den flacheren 
Verlauf der Kennlinien ron Aufangs- und Dauerströmen bewirkt, wie a. a. 0 . mehrfach beschrieben wurde.

In gleicher Weise ist die Kurve III zu deuten: Durch die 20 stündige Geißlerentladung wurde die Haut an den Sondenoberflächen abermals verstärkt. Sie bietet also den hindurchdringenden Elektronen oder Ionen einen abermals größeren Widerstand, wodurch sich einesteils der abermals flachere Verlauf der Kennlinien erklärt (die bier nicht wiedergegeben sind) und anderenteils die Fähigkeit der Sonden, Elektrizität aufzustapeln, so vermehrt worden ist, dab der angenähert wagrechte Teil der Kurve aus dem Beobachtungsbereich herausgerückt ist, so daB die Kurve III nur den linearen Anstieg zeigt, der, wie erwähnt, einer Kapazität von $1 / 80$ Mikrofarad entspricht, was auch mit der größeren Hautdicke im Einklang steht.

Ebenso stimmen mit diesen Annahmen die Beobachtungen der Funkenstöße überein, die bei einer unvorsichtigen $\mathrm{Er}$ höhung der Spannung eintreten. Ich möchte sie mit einem. Durchschlagen der Oberflächenhüute vergleichen oder etwa dadurch erklären, daß durch Joulesche Wärme die schlechtleitenden Schichten an gewissen Stellen zerstört werden. An so behandelten Sonden sind immer Stellen mit beschädigtem Metall zu bemerken, oft schmelzen die Sonden auch bei einer solchen Gelegenheit plötzlich ab. Dies tritt natürlich bei kräftigeren Schichten crst bei höheren Potentialdifferenzen auf als bei schwächeren. Ist aber z. B, eine ältere Schicht (III) an einer Stelle zerstört worden, so liegt hier das blanke Metall frei oder ist nur mit einer dünnen neuen Haut bedeckt, so daB sich nun die ganze Sonde so verhält, wie eine frisch geschmirgelte (I).

9. Es ist verlockend, aus den ballistischen Ausschlägen auf die Dicke der Schichten, die die kondensatorartigen Wirkungen hervorrufen, zu schließen. Das würde ohne Schwierigkeit möglich sein, wenn die Dielektrizitätskonstante der Sclichten bekannt wäre. Ich habe nun beobachtet, daß 14 Tage langes Liegen frisch geschmirgelter Sonden in der Zimmerluft den Typus der ballistischen Ausschläge nicht merklich beeinflußte und vermute daher, daB die wirksame Schicht nicht durch 
eine Haut adsorbierter Luft gebildet wird; auch Oxydschichten können nicht die Ursache der Erscheinungen sein, weil die Versuche auch wit Platin-Iridiumsonden gelingen. Es scheint mir hingegen wahrscheinlich, daß Kohlenwasserstoffe, herrührend von den Schmiermitteln, die zum Dichten dẹr Hähne rerwendet wurden, die ,Gashäute" verursachen. Diese Vermutung wird gestütat durch die Beobachtungen von A. Schuster, der besonders hohe "Polarisationsspannungen" in verschiedenen reinen Kohlenwasserstoffen fand, wie ich a. a. O. erwähnte. Danach müßten dio Kondensatorwirkungen vermieden oder mindestens geschwächt werden können, wenn das Gas durch tliissige Luft und andere Vorsichtsmabregeln sauber gehalten würde, Mittel, die mir hier nicht zur Verfügung stehen. Ich möchte also für die Schichten eine mittlere Dielektrizitätskonstante, wie sie für höhere Kohlenwasserstoffe gilt, annehmen: etwa 2,1 .

Aus den Beobachtungen, die a. a. $O$. besprochen und dort durch die Figg. 2-7 wiedergegeben sind, ist zu folgern, daB insbesondere die kathodische Sonde für die verschiedenen Polarisationserscheinungen verantwortlich zu machen ist. (Es ist dort auch [S. 21] kurz eine Hypothese umrissen, die das Zustandekommen der Schichten erklären soll; sie würe vielleicht noch dahin zu erweitern, daB von den verschiedenen Ionen des Entladungsrohres die schwereren Kohlenwasserstoffe am besten an den Sonden haften bleiben.) Demnach dürfte für die Berechnung der Sondenkapazität aus der geometrischen Form nur eine einzelne Sonde in Betracht zu ziehen sein.

Nun gilt für einen zylindrischen Kondensator, wie er einer einzelnen Sondenkapazität entspricht, die Formel:

$$
r=\frac{D}{2} \cdot \frac{l r}{a},
$$

worin $l$ die Länge, $r$ den Radius der Sonde, $D$ die Dielektrizitätskonstante und $a$ die Dicke der wirksamen Schicht bedeutet; setzt man hier die Werte $l=6 \mathrm{~cm}, r=0,5 \mathrm{~mm}, D=2,1$ und für $c$, den Kurven I-III der Fig. 2 entsprechend, der Reihe nach $1 / 10,1 / 20,1 / 60$. Mikrofarad ein, so ergeben sich die Dicken der Schichten der Reihe nach zu

$$
a=35,70,210 \mu \mu \text {. }
$$


184 E. Rumpf ther die Polarisation in der Geißlerentladung.

Diese Zahlen sind insofern recht befriedigend zu nennen, als man so in den Bereich der GröBen von Schichten kommt, die Farben dünner Blättchen liefern; denn für die erste Ordnung der Newtonschen Farben wird eine Luftschichtdicke von 100 bis $245 \mu \mu$ angegeben; dem entspricht (unter Annalıme eines Brechungsverhältnisses 1,5) für höhere Kohlenwasserstoffe eine Schichtdicke von

$$
67 \text { bis } 170 \mu \mu \text {. }
$$

Damit stimmt die Beobachtung vortrefflich uberein, denu an älteren Sonden, die einige Stunden der GeiBlerentladung ausgesetzt waren, sind stets deutlich solche "Anlauffarben" zu bemerken.

10. Als Ergebnis dieser Arbeit ist zusammenzufassen: Die an Querelektroden in der GeiBlerentladung auftretenden Polarisationserscheinungen werden im wesentlichen von Schichten verursacht, die sich an den Oberflächen dieser Elektroden ausbilden und eine kapazitative Wirkung haben.

Graz, Physikalisches Institut der Universität.

(Eingegangen 10. Dezember 1919.) 